\title{
Confirmation of the existence of the X17 particle
}

\author{
D. S. Firak ${ }^{2}$, A. J. Krasznahorkay ${ }^{1, *}$, M. Csatlós ${ }^{1}$, L. Csige ${ }^{1}$, J. Gulyás ${ }^{1}$, M. Koszta ${ }^{1}$, B. Szihalmi ${ }^{1}$, J. Timár ${ }^{1}$, Á. Nagy $^{1}$, \\ N. J. Sas ${ }^{2}$, and A. Krasznahorkay ${ }^{3}$ \\ ${ }^{1}$ Institute for Nuclear Research (Atomki) P.O. Box 51, H-4001 Debrecen, Hungary \\ ${ }^{2}$ University of Debrecen, 4010 Debrecen, PO Box 105, Hungary \\ ${ }^{3}$ CERN, Geneva, Switzerland and Institute for Nuclear Research (Atomki), P.O. Box 51, H-4001 Debrecen, Hungary
}

\begin{abstract}
In a 2016 paper, an anomaly in the internal pair creation on the $M 1$ transition depopulating the 18.15 MeV isoscalar $1^{+}$state on ${ }^{8} \mathrm{Be}$ was observed. This could be explained by the creation and subsequent decay of a new boson, with mass $m_{\mathrm{X}} c^{2}=16.70 \mathrm{MeV}$. Further experiments of the same transition with an improved and independent setup were performed, which constrained the mass of the X17 boson $\left(m_{\mathrm{X}} c^{2}\right)$ and its branching ratio relative to the $\gamma$-decay of the ${ }^{8} \mathrm{Be}$ excited state $\left(B_{\mathrm{X}}\right)$, to $m_{\mathrm{X}} c^{2}=17.01(16) \mathrm{MeV}$ and $B_{\mathrm{X}}=$ $6(1) \times 10^{-6}$, respectively. Using the latter setup, the $e^{+} e^{-}$pairs depopulating the $21 \mathrm{MeV} J^{\pi}=0^{-} \rightarrow 0^{+}$ transition in ${ }^{4} \mathrm{He}$ were investigated and a resonance in the angular correlation of the pairs was observed, which could be explained by the same X17 particle, with mass $m_{\mathrm{X}} c^{2}=16.98 \pm 0.16$ (stat) \pm 0.20 (syst) MeV.
\end{abstract}

\section{Introduction}

A recent measurement of the angular correlation of $e^{+} e^{-}$ pairs from the $18.15 \mathrm{MeV} J^{\pi}=1^{+} \rightarrow 0^{+} M 1$ transition of ${ }^{8} \mathrm{Be}$ revealed an anomalous peak-like enhancement relative to the internal pair creation (IPC) at large $e^{+} e^{-}$separation angles [1]. This was interpreted as the creation and subsequent decay of a new boson with a mass of $m_{\mathrm{X}} c^{2}=16.70 \pm 0.35$ (stat) \pm 0.5 (syst) MeV. Later experiments on the same transition observed the same particle, with mass $m_{X} c^{2}=17.01 \pm 0.16$ (stat) \pm 0.20 (syst) [2]

The possibility that the anomaly could be explained without a new particle, but within nuclear physics, with an improved model of the reaction or by introducing a nuclear transition form factor was explored by Zhang and Miller [3]. They were unable to explain the anomaly with the former approach, and obtained unrealistic form factors for the latter one.

The statistical significance of the beryllium anomaly observation and the possible relation of the X17 boson to the dark matter problem, and the fact that it might explain the $(\mathrm{g}-2)_{\mu}$ puzzle [4, 5], sparked interested from the theoretical and experimental particle and hadron physics community. Some of the recent possible explanations for the anomaly shall be discussed next.

Feng et al. [4, 6] further expanded on the idea of the new boson, analysing it as a protophobic vector gauge boson mediating a fifth force, with weak coupling to Standard Model (SM) particles. This model explains the data obtained from the beryllium anomaly and why in certain other experiments no contribution from the X17 was observed.

\footnotetext{
*e-mail: kraszna@atomki.hu
}

The protophobic nature of the X17 arises mostly from searches for $\pi_{0} \rightarrow Z^{\prime}+\gamma$ decay in the NA48/2 experiment [7]. The X17 was not observed in this experiment, which requires that the coupling of the X17 particle to the up and down quarks to be protophobic. This means that the charges $e \epsilon_{u}$ and $e \epsilon_{d}$ of the up and down quarks, written as multiple of the positron charge $e$, satisfy the relation $2 \epsilon_{u}+$ $\epsilon_{d} \leq 10^{3}$ [4, 6]. Many studies of such protophobic models were subsequently performed, including an extended two Higgs doublet model by Delle Rose and co-workers [8].

Delle Rose et al. [9] described the anomaly with a light $\mathrm{Z}_{0}$ bosonic state, arising from the $\mathrm{U}(1) 0$ symmetry breaking, with significant axial couplings so to evade low scale experimental constraints. They also showed how both spin- 0 and spin- 1 solutions are possible and describe the Beyond the Standard Model (BSM) that can accommodate these, including frameworks with either an enlarged Higgs, or gauge sector, or both.

Ellewanger and Moretti [10] made yet another explanation for the anomaly, using a light pseudoscalar particle. The X17 could be a $J^{\pi}=0^{-}$pseudoscalar particle, due to the quantum-numbers of the exited states and ground state of ${ }^{8} \mathrm{Be}$. In that case, they predicted that the branching ratio for the $17.6 \mathrm{MeV}$ transition should be about ten times smaller than the $18.15 \mathrm{MeV}$ one, which agrees with the experimental results.

In a recent experiment, the existence of the $\mathrm{X} 17$ boson was also observed on the $21 \mathrm{MeV}$ transition of ${ }^{4} \mathrm{He}$, which is also reported in this note. This reinforces the idea of new physics, by excluding the possibility of interference from decay channels from nearby energy levels. This is an important result, since a previous observation made by Boer et al. [11] of a possible light boson candidates seen from deviations from the expected IPC spectrum obtained 


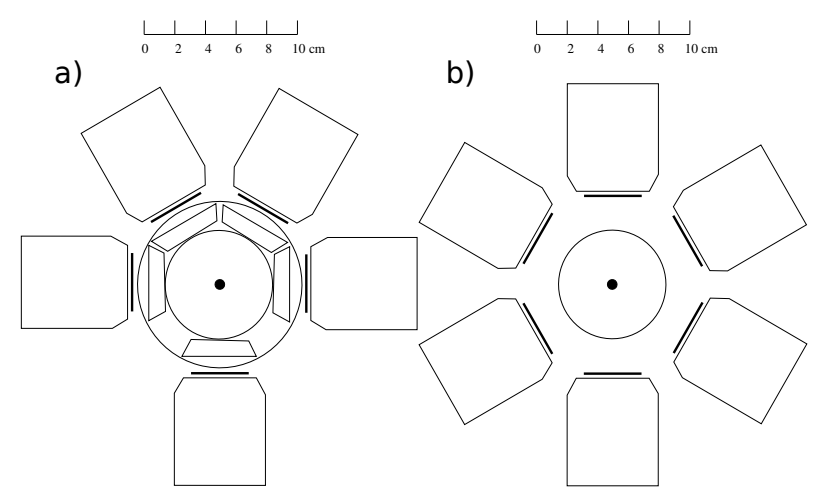

Figure 1. Comparison between the old and new setups. The previous setup (a) used 5 telescopes, each with a MWPC to gather the position of the particles and a thin scintillator in front of the main one to differentiate electrons and positrons from gammas. The new setup (b) consisted of 6 telescopes, and the MWPCs was replaced by DSSDs, which can be used for the particle identification, removing the need for the thin scintillators.

by the decay of a $17.6 \mathrm{MeV}$ excited state in ${ }^{8} \mathrm{Be}$, could be explained without new physics, but by considering some mixing from $E 1$ transitions from nearby energy levels to the explored $M 1$ transition (specifically, a $M 1+23 \%$ E1 mixed transition could explain Boer's results) [1]. Despite the beryllium anomaly described by Krasznahorkay et al. [1] being significantly different than Boer's (the latter being an excess instead of a bump), the false-alarm left the particle physics community sceptical of new a particle interpretations from similar experiments.

\section{Experiments}

The ${ }^{7} \operatorname{Li}(\mathrm{p}, \gamma){ }^{8} \mathrm{Be}$ reaction was used to populate the 17.6 $\mathrm{MeV}$ and $18.15 \mathrm{MeV}{ }^{8} \mathrm{Be}$ states, with proton energies of $E_{p}=441 \mathrm{keV}$ and $E_{p}=1030 \mathrm{keV}$. The experiment was performed on the $2 \mathrm{MV}$ Tandetron accelerator at MTA Atomki. A proton beam with a current of $1.0 \mu \mathrm{A}$ was impinged on a $15 \mu \mathrm{g} / \mathrm{cm}^{2} \mathrm{LiF}$ target for the $441 \mathrm{keV}$ resonance, and on a $300 \mu \mathrm{g} / \mathrm{cm}^{2} \mathrm{LiF}$ thick target evaporated onto $20 \mu \mathrm{g} / \mathrm{cm}^{2}$ carbon foils, for the $1030 \mathrm{keV}$ resonance. Given that the energy loss in the targets was of $9 \mathrm{keV}$ and $70 \mathrm{keV}$, respectively, the actual proton bombarding energy was set to $450 \mathrm{keV}$ and $1100 \mathrm{keV}$ [2].

In contrast to the previous experiment [1], a much thinner carbon backing was used, the number of telescopes was increased from 5 to 6, and the MWPC detectors were replaced by double sided silicon strip detectors (DSSDs), with a larger effective area. Those improvements, particularly the change in number and angle of telescopes, changed the efficiency for $e^{+} e^{-}$pair detections. The improved setup consisted of 6 telescopes on a plane perpendicular to the beam direction, each at $60^{\circ}$ to its neighbours. Each telescope contains a plastic scintillator, with dimensions of $82 \times 86 \times 80 \mathrm{~mm}^{3}$, and a $50 \times 50 \mathrm{~mm}^{2}$ DSSD with 16 strips for each direction. The target was placed in a carbon fibre vacuum chamber, with $1 \mathrm{~mm}$ thick walls, in the centre of the detection system.
To monitor $\gamma$-rays produced from the decay of the 18.15 MeV state, a $\epsilon_{\text {rel }}=100 \%$ High Purity (HP) germanium detector was placed $25 \mathrm{~cm}$ away from the target.

The ${ }^{3} \mathrm{H}(\mathrm{p}, \gamma)^{4} \mathrm{He}$ reaction was used to populate the broad second excited state in ${ }^{4} \mathrm{He}\left(E_{x}=21.1 \mathrm{MeV}\right.$, $\left.\Gamma=0.84 \mathrm{MeV}, J^{\pi}=0^{-}\right)$, with a proton energy of $E_{p}=0.900 \mathrm{MeV}$, which is below the $1.018 \mathrm{MeV}$ threshold for the $(\mathrm{p}, \mathrm{n})$ reaction. The first excited state in ${ }^{4} \mathrm{He}$ $\left(E_{x}=20.21 \mathrm{MeV}, \Gamma=0.50 \mathrm{MeV}, J^{\pi}=0^{+}\right)$overlaps with the second, and it de-excites via an $E O$ transition.

For the ${ }^{3} \mathrm{H}(\mathrm{p}, \gamma){ }^{4} \mathrm{He}$ reaction, the target was a tritated titanium disk $3.0 \mathrm{mg} / \mathrm{cm}^{2}$ thick, evaporated onto a $0.4 \mathrm{~mm}$ tick Mo disk. The concentration of tritium atoms was $2.66 \times 10^{20}$ atoms $/ \mathrm{cm}^{2}$. To avoid evaporation of tritium, the target was kept at a liquid nitrogen temperature.

For all experiments, the energy calibration was obtained from the $6.05 \mathrm{MeV}$ IPC $E O$ transition from the ${ }^{19} \mathrm{~F}\left(\mathrm{p}, \alpha+e^{+} e^{-}\right){ }^{16} \mathrm{O}$ reaction. Any non-linearity effects, due to the signal amplification or otherwise, would be seen from the $17.6 \mathrm{MeV}$ transitions from the $\mathrm{Li}(\mathrm{p}, \gamma) \mathrm{Be}$ reaction.

The angular efficiency of the setup was determined by sampling neighbouring events from the same dataset, guaranteeing no correlation between them. The efficiency is then used to provide a setup independent result. Reference [12] describes the previous setup, with 5 telescopes (seen on Fig. 1. (a)) and a set up similar to the one used on the current experiments (seen on Fig. 1](b)). The efficiencies for pair detection from both setup geometries differ significantly, hence the results with the new one can be considered as an independent measurement.

\section{Experimental results: ${ }^{8} \mathrm{Be}$ experiment}

In the ${ }^{8} \mathrm{Be}$ experiments, both the $18.15 \mathrm{MeV}$ and the 17.6 MeV transition were observed. While no signal enhancement was observed for the 17.6 MeV transition on either experiments, it was used to check for non-linearity effects during the energy calibration. Figure 2 shows the resulting sum energy and angular correlation spectra for the improved experimental setup. It is in agreement with the previous experiment [1], the $M 1$ transition follows theoretical predictions, without the contribution of the X17 on the $17.6 \mathrm{MeV}$ transition.

Figure 3 shows the results for the $18.15 \mathrm{MeV}^{8} \mathrm{Be}$ transition. In red dots with error bars the current results [2] are shown, while in blue the previous results are shown [1]. There is a good agreement between both experiments.

\subsection{Function fitting}

The $e^{+} e^{-}$background angular distribution is modelled by an exponentially decreasing distribution, and the boson is modelled after simulations of a boson decaying to $e^{+} e^{-}$pairs.

The fit was performed using RooFit [13], with the following distribution function:

$P D F\left(e^{+} e^{-}\right)=N_{b k g d} * P D F(I P C)+N_{s i g} * P D F($ signal $)$ 

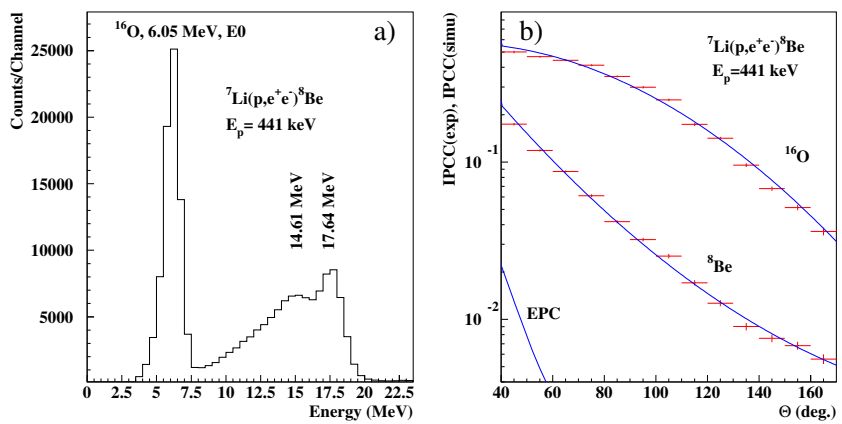

Figure 2. Energy sum spectrum (a) and angular correlation (b) of the $e^{+} e^{-}$pairs from the $17.6 \mathrm{MeV}$ transition. Full blue curve shows the simulated results, and red points with error bars shows the experimental results

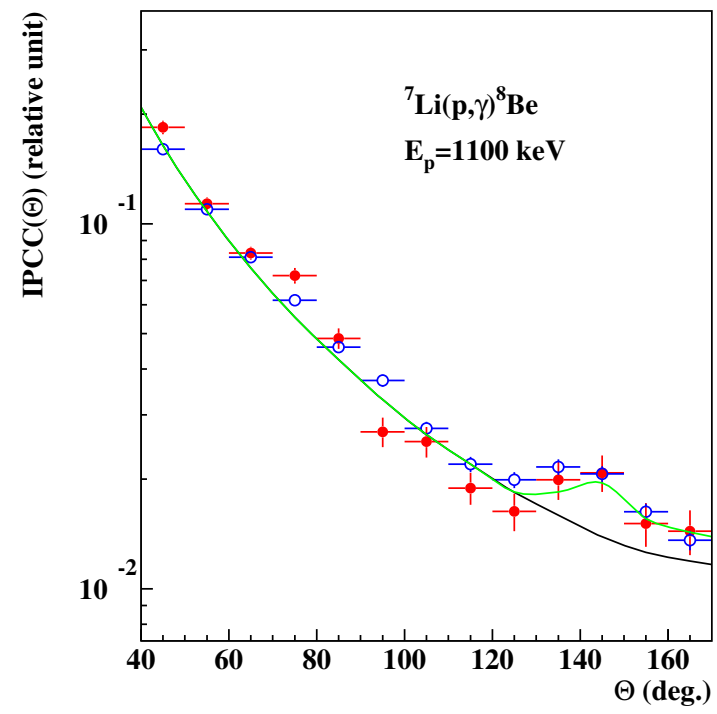

Figure 3. Results from the ${ }^{8} \mathrm{Be}$. Background, shown by the solid black line, is estimated by a fourth order exponential polynomial. The green solid line shows simulation results, which include the decay of the boson. Red dots with error bars shows the results from the latest experiment [2] for the ${ }^{8} \mathrm{Be} 18.15 \mathrm{MeV}$ transition, while blue circles with error bars show the previous results [1]

where $N_{b k g d}$ and $N_{s i g}$ are the number of background and signal events, respectively.

To model the signal, a two dimensional distribution was constructed, with mass and opening angle dependencies. The mass dependency was obtained from linear interpolation of the $e^{+} e^{-}$angular distribution, simulated for discrete particle masses.

With the PDF described in Equation 1 . fits were performed to determine the $N_{b k g d}$ and $N_{\text {sig }}$, by fixing a mass on the signal PDF. The best fitted values were taken from this method. To obtain the mass precisely, a fit was made with the mass as a fit parameter. With the results, the branching ratio relative to the $\gamma$-decay was calculated for the best fit.

The results published in [1] are $m_{\mathrm{X}} c^{2}=16.70(51) \mathrm{MeV}$ and $B_{\mathrm{X}}=5.8 \times 10^{-6}$, with $6.8 \sigma$ significance. The same data fitted with the method listed above yields $m_{\mathrm{X}} c^{2}=16.86(6) \mathrm{MeV}$ and $B_{\mathrm{X}}=6.8(10) \times 10^{-6}$, with $7.37 \sigma$ significance. The new experiment [2] resulted a mass of $m_{\mathrm{X}} c^{2}=17.17(7) \mathrm{MeV}$ and a relative branching ratio of $4.7(21) \times 10^{-6}$, with $4.90 \sigma$ significance. The difference between the obtained mass of the X17 particle in each dataset are larger than the statistical error. This can be due to the uncertainty of the beam position on the target, or some misalignment of the detectors, which affects the determination of the position of the hits relative to the target, therefore skewing the angular correlation between the $e^{+} e^{-}$pairs.

By averaging the results for the ${ }^{8} \mathrm{Be}$ experiments, the mass and relative branching ratio were determined to be $m_{\mathrm{X}} c^{2}=17.01(16)$ and $B_{\mathrm{X}}=6(1) \times 10^{-6}$.

\section{Experimental results: ${ }^{4} \mathrm{He}$ Experiment}

The expected angular correlation for $e^{+} e^{-}$pairs from the $\mathrm{X} 17$ boson in the decay of the $21.0 \mathrm{MeV}{ }^{4} \mathrm{He}$ state is at around $110^{\circ}$, instead of the $140^{\circ}$ observed on the ${ }^{8} \mathrm{Be}$ experiment, due to the higher energy of the ${ }^{4} \mathrm{He}$ transition. This higher energy results in a larger kinetic boost for the $\mathrm{X} 17$, which yields lower opening angles between the decay products of the X17.

Since the expected angular correlation for the $e^{+} e^{-}$pairs for the boson is peaked around $110^{\circ}$, the energy sum spectra was also taken for pairs $60^{\circ}$ and $120^{\circ}$ apart. While the telescopes at $120^{\circ}$ should contain some enhancement from the decay of the boson, the telescopes at $60^{\circ}$ should provide a background, which can then be used to determine a signal region for the transition. As seen on Fig. 4, when taking the difference of those energy sum spectra, it becomes clear that the signal region is $19.5 \mathrm{MeV} \leq E_{\text {total }} \leq 22.0 \mathrm{MeV}$

Figure 5 shows the angular correlation results for the previously mentioned ${ }^{4} \mathrm{He}$ transition. The $e^{+} e^{-}$pairs were gated by the energy sum on the signal region for the transition (between $19.5 \mathrm{MeV}$ and $22.0 \mathrm{MeV}$ ), and with an asymmetry parameter, defined in Ref. [1], such that $|y| \leq 0.5$.

The peak appears at $115^{\circ}$, which is consistent with the X17 interpretation, with mass of $m_{\mathrm{X}} c^{2}=16.98 \pm$ 0.16 (stat) \pm 0.20 (syst) $\mathrm{MeV}$.

\section{Future experiments}

In the coming years, several independent particle physics experiments will probe the same parameter space of the X17 boson. Their results will be fundamental in determining if the existence of such particle is true or not. Some of these experiments will be briefly discussed. Additional discussion can be found in Ref. [6].

The NA64 experiment at CERN searched with a 100 $\mathrm{GeV} / \mathrm{c} e^{-}$beam for a hypothetical boson with mass $m_{\mathrm{X}} c^{2}=$ $16.7 \mathrm{MeV}$, near the proposed mass of the X17. It covers most, but not all, of the allowed $\epsilon_{e}$ parameter space for protophobic bosons [14].

The DarkLight experiment, which will search for dark photons in the $10 \mathrm{MeV} / \mathrm{c}^{2}$ to $100 \mathrm{MeV} / \mathrm{c}^{2}$ energy range, is 

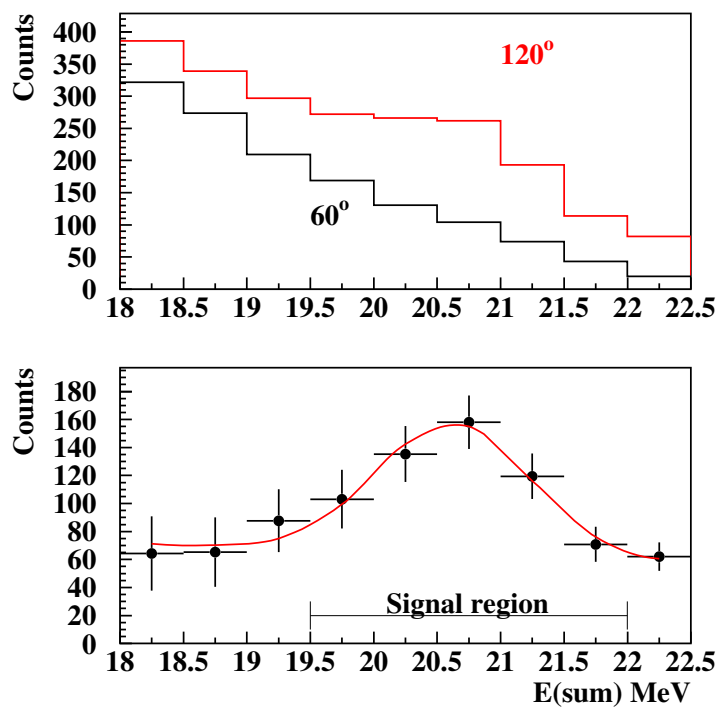

Figure 4. Top figure: summed energy spectra from different telescope pair angles: for telescopes $120^{\circ}$ apart is shown in red, and in black for telescopes $60^{\circ}$ apart, which are used as a background measurement. Bottom figure: measured energy sum from $e^{+} e^{-}$pairs originated from the $21 \mathrm{MeV}{ }^{4} \mathrm{He}$ state decay; the background coming from the target was subtracted, but not the constant one caused by the cosmic rays

projected to cover most of the allowed $\epsilon_{e}$ parameter space for protophobic boson [15]. The experiment aims to produce dark photons by scattering $e^{-}$off a hydrogen gas target. A proof-of-principle measurement is currently being done [16].

The MESA experiment, similarly to the DarkLight, will be searching for dark photon with electron scattering of hydrogen gas. The explored mass range of MESA will be between 10 and $40 \mathrm{MeV} / \mathrm{c}^{2}$ [17].

The BESIII experiment currently contains the largest dataset of $\mathrm{J} / \psi$ events (around $10^{10}$ events). Jiang, Yang and Qiao [18] proposed that an analysis of the current dataset for new gauge bosons would be possible, expecting around $10^{3}$ scalar, $Z_{0}$-like bosons under specific conditions.

The ForwArd Search ExpeRiment (FASER) [19] at LHC is set to search for light, weakly interacting particles, such as axiom-like particles [20-23], with a detector placed in the forward regions of ATLAS.

The search for light gauge boson was proposed in $e^{+} e^{-}$collision experiments or $e^{+}$beam dump experiments, namely the aforementioned BESIII experiment [18], the BaBar experiment [24], the PADME experiment [25], and the KLOE-2 experiment [26].

The PADME experiment is running until the end of 2019, and will be moved to Cornell and/or JLAB to get higher intensity positron beams [27,-30].

Within the large scope of the KLOE-2 experiment, [31] realised with the improved DAФNE- $2 \phi$-factory, there is a search for $e^{+} e^{-} \rightarrow \gamma\left(X \rightarrow e^{+} e^{-}\right)$events [26].

Experiments exploring other high-energy nuclear transitions would also shed light on the anomaly. Previous

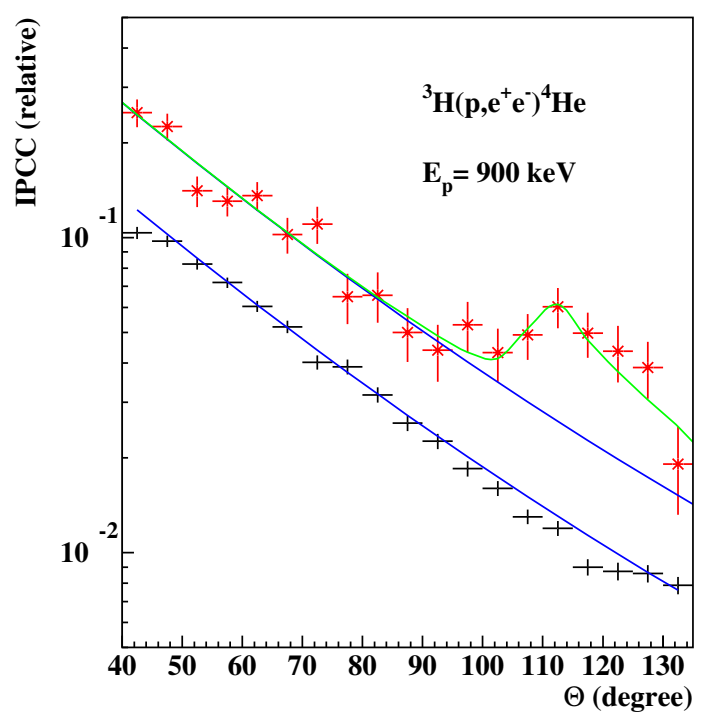

Figure 5. Angular correlation for $e^{+} e^{-}$pairs detected from the $21 \mathrm{MeV}{ }^{4} \mathrm{He}$ transition. The background, taken from outside the signal region, is shown in black dots, with error bars. A fourth order exponential polynomial is used to fit the background. The result is shown with a solid blue line. The result for the signal region is seen in red asterisks, with error bars. The solid green curve shows simulation results, which take the decay of the X17 boson into consideration.

experiments performed in the 1970s explored such highenergy transitions [32, 33], but without the required production cross section and branching ratio to observe deviations on IPC.

\section{Conclusions}

The anomalous angular correlation observed on the original experiment was reproduced using the new independent setup with the same $18.5 \mathrm{MeV}$ transition from the ${ }^{7} \mathrm{Li}(\mathrm{p}, \gamma){ }^{8} \mathrm{Be}$ reaction. A signal was also observed on the $21.0 \mathrm{MeV}$ transition of ${ }^{4} \mathrm{He}$. The ${ }^{4} \mathrm{He}$ signal can be explained by the same new X17 particle, with mass $m_{\mathrm{X}} c^{2}=16.98 \pm 0.16$ (stat) \pm 0.20 (syst) $\mathrm{MeV}$, which agrees with the mass range obtained from the ${ }^{8} \mathrm{Be}$ experiments $\left(m_{\mathrm{X}} c^{2}=17.01 \pm 0.16 \mathrm{MeV}\right)$.

The observation of a similar anomalous internal pair creation on the $21 \mathrm{MeV}$ transition of ${ }^{4} \mathrm{He}$ is strong evidence for new physics, since it excludes the possibility of interference from other decay channels from excited states near $18.5 \mathrm{MeV}$ present on the ${ }^{8} \mathrm{Be}$ case.

Many experiments in the coming years will be looking directly at the possibility of a new gauge boson, or indirectly, by probing the same parameter space as the X17. This will likely determine the existence of such particle, and constrain its properties.

The beryllium anomaly observed in 2016 shows that nuclear physics can be taken as a relatively cheap laboratory for particle physics, and the many unsolved problems of physics, which may be partially or fully explained with the existence of weakly interacting light particles, are an 
incentive to keep realising such experiments as the ones described in this note.

\section{Acknowledgements}

This work has been supported by the Hungarian NKFI Foundation No. K124810, by the GINOP-2.3.3-15-201600034 and by the János Bolyai Research Fellowship of the Hungarian Academy of Sciences (L. Csige).

\section{References}

[1] A. J. Krasznahorkay, M. Csatlós, L. Csige et al., Phys. Rev. Lett. 116, 042501 (2016).

[2] A. J. Krasznahorkay, M. Csatlós, L. Csige et al., Acta Phys. Pol. B 50, 675 (2019).

[3] X. Zhang and G. A. Miller, Phys. Lett. B 773, 159 (2017).

[4] J. L. Feng, B. Fornal, I. Galon et al., Phys. Rev. Lett. 117, 071803 (2016).

[5] M. Pospelov, Phys. Rev. D 80, 095002 (2009).

[6] J. L. Feng, B. Fornal, I. Galon et al., Phys. Rev. D 95, 035017 (2017).

[7] J. R. Batley, G. Kalmus, C. Lazzeroni et al., Phys. Lett. B 746, 178 (2015).

[8] L. D. Rose, S. Khalil, and S. Moretti, Phys. Rev. D 96, 115024 (2017).

[9] L. D. Rose, S. Khalil, S. J. D. King et al., Phys. Rev. D 99, 055022 (2019).

[10] U. Ellwanger and S. Moretti, J. High Energy Phys. 11, 39 (2016).

[11] F. W. N. de Boer, O. Fröhlich, K. E. Stiebing et al., Phys. Lett. B 388, 235 (1996).

[12] J. Gulyás, T. J. Ketel, A. J. Krasznahorkay et al., Nucl. Instrum. Meth. A 808, 21 (2016).

[13] W. Verkerke and D. P. Wouter, EPJ Web Conf. 4, 02005 (2010).

[14] D. Banerjee, V. E. Burtsev, A. G. Chumakov et al., Phys. Rev. Lett. 120, 231802 (2018).
[15] J. Balewski, J. Bernauer, J. Bessuille et al., arXiv eprints, arXiv:1412.4717 (2014).

[16] Y. Wang, R. Corliss, R. G. Milner et al., Nucl. Instrum. Meth. A 935, 1 (2019).

[17] T. Beranek, H. Merkel, and M. Vanderhaeghen, Phys. Rev D 88, 015032 (2013).

[18] J. Jian, H. Yang, and C.-F. Qiao, Eur. Phys. J. C 79, 404 (2019).

[19] J. L. Feng, I. Galon, F. Kling et al., Phys. Rev. D 98, 055021 (2018).

[20] A. Ariga, T. Ariga, J. Boyd et al., arXiv e-prints, arXiv: 1901.04468 (2019).

[21] A. Ariga, T. Ariga, J. Boyd et al., arXiv e-prints, arXiv:1811.12522 (2019).

[22] A. Ariga, T. Ariga, J. Boyd et al., arXiv e-prints, arXiv:1812.09139 (2019).

[23] A. Ariga, T. Ariga, J. Boyd et al., Phys. Rev. D 99, 095011 (2019).

[24] L. -B. Chen, Y. Liang, and C.-F. Qiao, arXiv e-prints, arXiv:1607.03970 (2016).

[25] M. Raggi, V. Kozhuharov, and P. Valente, EPJ Web Conf. 96, 01025 (2015).

[26] A. Anastasi, D. Babusci, G. Bencivenni et al., Phys. Rev. Lett. 113 no. 20, 201801 (2014).

[27] C. Taruggi on behalf of the PADME Collaboration, Frascati Physics Series 67, 17 (2018).

[28] S. Spagnolo on behalf of the PADME Collaboration, J. Phys. Conf. Ser. 1137, 012043 (2018).

[29] G. Piperno on behalf of the PADME Collaboration, J. Phys. Conf. Ser. 1162, 012031 (2019).

[30] V. Kozhuharov, EPJ Web Conf. 212, 06001 (2019).

[31] G. Amelino-Camelia, F. Archilli, D. Babusci et al., Eur. Phys. J. C 68, 619 (2010).

[32] S. C. Ling and S. L. Blatt, Nucl. Phys. A 174, 375 (1971).

[33] K. Subotic, B. Lalovic, and B. Stepancic, Nucl. Phys. A 296, 141 (1978). 